\title{
Synthesis of Methyl (E)-2-Cyanomethylcinnamates Derived from Baylis-Hillman Acetates and Conversion into Several 4-Hydroxy-2-naphthoic Acids and Benzylidenesuccinimides
}

\author{
Wan Pyo Hong, Hee Nam Lim, Hong Woo Park, and Kee-Jung Lee* \\ Department of Chemical Engineering, Hanyang University, Seoul 133-791, Korea. *E-mail: leekj@hanyang.ac.kr \\ Received February 22, 2005
}

Key Words : Baylis-Hillman reaction, Methyl 2-cyanomethylcinnamate, 4-Hydroxy-2-naphthoic acid, Benzylidenesuccinimide

The Baylis-Hillman $(\mathrm{BH})$ reaction $^{1}$ has been developed enormously over the past few years due to its wide applicability towards formation of multifunctional derivatives, ${ }^{2}$ heterocycles ${ }^{3}$ and natural products. ${ }^{4}$ Aliphatic nitriles are potentially useful building block in organic synthesis due to the electron-withdrawing nature associated with the cyano group and the conversion of the cyano group into other functionalities. ${ }^{5}$

Much attention has recently been focussed on the $S_{N} 2^{\prime}$ nucleophilic substitution of the Baylis-Hillman acetates. ${ }^{6}$ Among them only limited approaches to the cyanation of $\mathrm{BH}$ adducts are known in the literature. The one method is to access ethyl 3-cyano-2-methylcinnamates through DABCO assisted the successive $\mathrm{S}_{N} 2^{\prime}-\mathrm{S}_{N} 2^{\prime}$ reaction of $\mathrm{BH}$ acetates with $\mathrm{KCN}^{7}$ and the other methods are Michael addition of $\mathrm{KCN}$ with $O$-t-butyldimethylsilyl BH adduct of piperonal, ${ }^{8}$ and several $\mathrm{S}_{N} 2^{\prime}$ reaction of $\mathrm{BH}$ acetates of 2-azidobenzaldehyde with $\mathrm{KCN}$ by us. ${ }^{3 \mathrm{~b}}$

As part of our continuing studies towards development of the BH chemistry, ${ }^{9}$ we desired to have the cyano group at the allylic position of the 3-aryl-2-propenoates. In principle, such nitrile compounds might be extended further towards the building of naphthalene and succinimide derivatives.

Treatment of $\mathrm{BH}$ acetate $1 \mathrm{a}$ with $\mathrm{KCN}$ in $\mathrm{DMSO} / \mathrm{H}_{2} \mathrm{O}$ at room temperature for $1 \mathrm{~h}$ afforded 2-cyanomethylcinnamic acid methyl ester (2a) in $74 \%$ yield. This success led us to transform a variety of methyl 3-acetoxy-3-aryl-2-methylenepropanoates 1b-i into methyl (E)-3-aryl-2-cyanomethyl2-propenoates $\mathbf{2 b}-\mathbf{i}$ stereoselectively under the similar reaction conditions (Scheme 1, Table 1). The E-geometry of the olefinic bond was established on the basis of ${ }^{1} \mathrm{H}$ NMR data of the vinyl peaks appeared at 7.70-8.03 ppm, which were well coincident with the reported data of similar<smiles>[R]C(OC(C)=O)C(=C)C(=O)O[Na]</smiles>
1<smiles>[R]C=C(CC#N)COC</smiles>

2
For $\mathrm{R}$ see Table 1 compounds. $^{3 \mathrm{~b}}$

To confirm the synthetic efficacy of (E)-3-aryl-2-cyanomethyl-2-propenoates 2, we prepared several known 4hydroxy-2-naphthoic acids $4 \mathbf{4 a - c}$ by dehydration of $(E)$ benzylidenesuccinic acids 3a-c with conc. $\mathrm{H}_{2} \mathrm{SO}_{4}$ at room temperature, ${ }^{10}$ which were obtained from the hydrolysis of 2a-c under basic conditions. Also, we examined the feasibility of transforming several cyanomethylcinnamates 2a-c into the corresponding benzylidenesuccinimides 8a-c by using nitrile group transformation strategy. Several methods for the synthesis of benzylidenesuccinimides involve the Wittig reaction of triphenylphosphoranylidenesuccinimides with aromatic aldehydes ${ }^{11}$ and the Stobbe condensation route. ${ }^{12}$

In our work we found that use of acetic acid in the presence of $\mathrm{FeCl}_{3}{ }^{13}$ at reflux temperature led to the desired benzylidenesuccinimides in $40-45 \%$ yields. The plausible mechanism for the formation of imide 8a-c can be thought as shown in $\mathrm{Scheme} 2 . \mathrm{FeCl}_{3}$ makes the nitrile group more electrophilic, then subsequent nucleophilic addition of acetic acid to the cyano group generates imidate $\mathbf{6}$, which undergoes formation of lactam 7 , and cleavage of acetyl group by methanol gives succinimide $\mathbf{8}$.

In summary, a simple synthesis of methyl 2-cyanomethylcinnamates from readily available Baylis-Hillman acetates and conversion to the several naphthalene and benzylidensuccinimide derivatives is disclosed.

\section{Experimental Section}

Silica gel 60 (70-230 mesh ASTM) used for column Table 1. Methyl (E)-3-Aryl-2-cyanomethyl-2-propenoates 2

\begin{tabular}{clccc}
\hline Reactant & \multicolumn{1}{c}{$\mathrm{R}$} & Product & Yield $(\%)$ & $\mathrm{mp}\left({ }^{\circ} \mathrm{C}\right)$ \\
\hline $\mathbf{1 a}$ & $\mathrm{C}_{6} \mathrm{H}_{5}$ & $\mathbf{2 a}$ & 74 & oil \\
$\mathbf{1 b}$ & $4-\mathrm{ClC}_{6} \mathrm{H}_{4}$ & $\mathbf{2 b}$ & 57 & $85-87$ \\
$\mathbf{1 c}$ & $4-\mathrm{MeOC}_{6} \mathrm{H}_{4}$ & $\mathbf{2 c}$ & 69 & $64-65$ \\
$\mathbf{1 d}$ & $4-\mathrm{AcNHC}_{6} \mathrm{H}_{4}$ & $\mathbf{2 d}$ & 64 & $163-165$ \\
$\mathbf{1 e}$ & $2-\mathrm{ClC}_{6} \mathrm{H}_{4}$ & $\mathbf{2 e}$ & 79 & $83-85$ \\
$\mathbf{1 f}$ & $3-\mathrm{O}_{2} \mathrm{NC}_{6} \mathrm{H}_{4}$ & $\mathbf{2 f}$ & 33 & $88-90$ \\
$\mathbf{1 g}$ & $2,6-\mathrm{Cl}_{2} \mathrm{C}_{6} \mathrm{H}_{3}$ & $\mathbf{2 g}$ & 65 & $102-104$ \\
$\mathbf{1 h}$ & $3,4-\mathrm{MeOC}_{6} \mathrm{H}_{3}$ & $\mathbf{2 h}$ & 87 & $97-99$ \\
$\mathbf{1 i}$ & $2,6-\mathrm{Cl}_{2}-3-\mathrm{O}_{2} \mathrm{NC}_{6} \mathrm{H}_{2}$ & $\mathbf{2 i}$ & 27 & $80-82$ \\
\hline
\end{tabular}


<smiles>[R]C(=O)/C(=C/c1ccc([R])cc1)CC#N</smiles>

2<smiles></smiles>

5<smiles>[R]c1ccc(/C=C2\C=C(OC(C)=O)NC2=O)cc1</smiles>

$7 b$

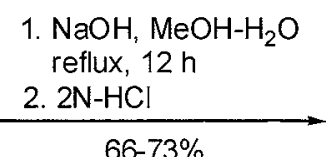

HOAC
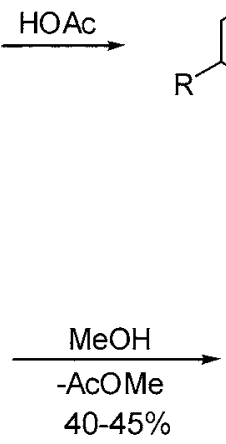

$40-45 \%$<smiles>[R]c1ccc(/C=C(\CC(=O)O)C(=O)O)cc1</smiles>

3

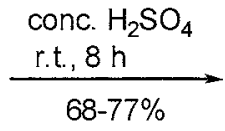<smiles>[R]c1ccc2cc(C(=O)O)cc(O)c2c1</smiles>

4<smiles>[R]c1ccc(/C=C(\CC(=N)OC(C)=O)C(=[O+])OC)cc1</smiles>

6<smiles>[R]c1ccc(/C=C2\CC(=O)NC2=O)cc1</smiles>

8<smiles>[R]c1ccc(/C=C2\CC(OC(C)=O)=NC2=O)cc1</smiles>

$7 a$

Scheme 2

chromatography was supplied by E. Merck. Analytical thin layer chromatography (TLC) was carried out on Merck silica gel 60 F254 TLC plates. Melting points were taken using an Electrothermal melting point apparatus and are uncorrected. Infrared spectra were recorded on a Nicolet Magna 550 FTIR spectrometer. The ${ }^{1} \mathrm{H}$ and ${ }^{13} \mathrm{C}$ NMR spectra were measured on a Gemini 300 spectrometer using $\mathrm{CDCl}_{3}$ or DMSO- $\mathrm{d}_{6}$. All chemical shifts are reported in ppm relative to TMS and coupling constants ( $\mathrm{J}$ ) are expressed in $\mathrm{Hz}$.

Typical Procedure for the Preparation Methyl $(E)-3$ Aryl-2-cyanomethyl-2-propenoate 2a: To a stirred solution of $1 \mathbf{a}(234 \mathrm{mg}, 1.0 \mathrm{mmol})$ in 1 : 1 DMSO- $\mathrm{H}_{2} \mathrm{O}(6 \mathrm{~mL})$ was added $\mathrm{KCN}$ (98 mg, $1.5 \mathrm{mmol}$ ) at room temperature. After stirring at the same temperature for $1 \mathrm{~h}$, the reaction mixture was diluted with water $(10 \mathrm{~mL})$ and extracted with $\mathrm{CH}_{2} \mathrm{Cl}_{2}(3 \times 15 \mathrm{~mL})$. The combined organic layers were dried over anhydrous $\mathrm{MgSO}_{4}$ and the solvent was evaporated in vacuo. The resulting mixture was chromatographed on silica gel eluting with hexane/EtOAc $(6: 1)$ to afford $149 \mathrm{mg}(74 \%)$ of $\mathbf{2 a}$ as a liquid; IR (neat) 2250, 1710, $1637 \mathrm{~cm}^{-1} ;{ }^{1} \mathrm{H}$ NMR $\left(\mathrm{CDCl}_{3}\right) \delta 3.54(\mathrm{~s}, 2 \mathrm{H}), 3.90(\mathrm{~s}, 3 \mathrm{H})$, 7.39-7.51 (m, 5H), $7.98(\mathrm{~s}, 1 \mathrm{H}) ;{ }^{13} \mathrm{C} \mathrm{NMR}\left(\mathrm{CDCl}_{3}\right)$ 16.92, 52.72, 117.31, 121.81, 129.03, 129.69, 130.66, 133.69, 144.02, 166.16 .

The spectroscopic data of the synthesized compounds are as follows.

2b: $135 \mathrm{mg}(57 \%)$; $\mathrm{mp} 85-87{ }^{\circ} \mathrm{C}$; IR (KBr) 2253, 1713, $1642 \mathrm{~cm}^{-1} ;{ }^{1} \mathrm{H} \mathrm{NMR}\left(\mathrm{CDCl}_{3}\right) \delta 3.51(\mathrm{~s}, 2 \mathrm{H}), 3.90(\mathrm{~s}, 3 \mathrm{H})$, $7.34(\mathrm{~d}, 2 \mathrm{H}, J=8.5 \mathrm{~Hz}), 7.46(\mathrm{~d}, 2 \mathrm{H}, J=8.5 \mathrm{~Hz}), 7.91(\mathrm{~s}$, $1 \mathrm{H}) ;{ }^{13} \mathrm{C} \mathrm{NMR}\left(\mathrm{CDCl}_{3}\right) 16.70,52.72,116.90,122.48$, 129.27, 130.40, 132.09, 135.92, 142.53, 165.85 .

2c: $159 \mathrm{mg}(69 \%)$; $\mathrm{mp} 64-65^{\circ} \mathrm{C}$; IR (KBr) 2245, 1711,
$1628 \mathrm{~cm}^{-1} ;{ }^{1} \mathrm{H}$ NMR $\left(\mathrm{CDCl}_{3}\right) \delta 3.57(\mathrm{~s}, 2 \mathrm{H}), 3.86(\mathrm{~s}, 3 \mathrm{H})$, 3.88 (s, 3H), 6.99 (d, 2H, J=8.6 Hz), 7.39 (d, 2H, $J=8.6 \mathrm{~Hz})$, $7.91(\mathrm{~s}, 1 \mathrm{H}) ;{ }^{13} \mathrm{C} \mathrm{NMR}\left(\mathrm{CDCl}_{3}\right) 16.92,52.60,55.37,114.46$, $117.47,119.27,126.07,131.27,143.65,160.85,166.52$.

2d: $166 \mathrm{mg}(64 \%)$; mp 163-165 ${ }^{\circ} \mathrm{C}$; IR (KBr) 3424, 2248, 1693, $1669 \mathrm{~cm}^{-1} ;{ }^{1} \mathrm{H} \mathrm{NMR}\left(\mathrm{CDCl}_{3}\right) \delta 2.21(\mathrm{~s}, 3 \mathrm{H}), 3.56(\mathrm{~s}$, 2H), $3.90(\mathrm{~s}, 3 \mathrm{H}), 7.39$ (d, 2H, $J=8.6 \mathrm{~Hz}), 7.58$ (br s, 1H), $7.64(\mathrm{~d}, 2 \mathrm{H}, J=8.6 \mathrm{~Hz}), 7.91(\mathrm{~s}, 1 \mathrm{H}) ;{ }^{13} \mathrm{C} \mathrm{NMR}\left(\mathrm{CDCl}_{3}\right)$ 16.96, 24.68, 52.75, 117.41, 119.76, 120.56, 129.26, 130.45, 139.47, 143.38, 166.36, 168.58 .

2e: $186 \mathrm{mg}(79 \%)$; $\mathrm{mp} \mathrm{83-85}{ }^{\circ} \mathrm{C}$; IR (KBr) 2251, 1716, $1635 \mathrm{~cm}^{-1} ;{ }^{1} \mathrm{H}$ NMR $\left(\mathrm{CDCl}_{3}\right) \delta 3.42(\mathrm{~s}, 2 \mathrm{H}), 3.92(\mathrm{~s}, 3 \mathrm{H})$, 7.37-7.40 (m, 3H), 7.46-7.50 (m, 1H), $8.03(\mathrm{~s}, 1 \mathrm{H}) ;{ }^{13} \mathrm{C}$ NMR $\left(\mathrm{CDCl}_{3}\right)$ 17.04, 52.80, 116.94, 123.95, 127.13, 129.59, 129.93, 130.83, 132.35, 133.99, 141.13, 165.53.

2f: $81 \mathrm{mg}(33 \%)$; $\mathrm{mp} 88-90{ }^{\circ} \mathrm{C}$; IR (KBr) 2251, 1709, $1638,1530,1355 \mathrm{~cm}^{-1} ;{ }^{1} \mathrm{H} \mathrm{NMR}\left(\mathrm{CDCl}_{3}\right) \delta 3.53(\mathrm{~s}, 2 \mathrm{H})$, 3.94 (s, 3H), 7.70-7.76 (m, 2H), $8.00(\mathrm{~s}, 1 \mathrm{H}), 8.24-8.32(\mathrm{~m}$, $2 \mathrm{H}) ;{ }^{13} \mathrm{C} \mathrm{NMR}\left(\mathrm{CDCl}_{3}\right)$ 16.63, 52.97, 116.36, 123.78, 124.13, 124.69, 130.17, 134.38, 135.22, 140.95, 148.41, 165.33.

2g: $175 \mathrm{mg}(65 \%)$; mp 102-104 ${ }^{\circ} \mathrm{C}$; IR (KBr) 2256, 1721, $1650 \mathrm{~cm}^{-1} ;{ }^{1} \mathrm{H} \mathrm{NMR}\left(\mathrm{CDCl}_{3}\right) \delta 3.26(\mathrm{~s}, 2 \mathrm{H}), 3.94(\mathrm{~s}, 3 \mathrm{H})$, 7.28-7.42 (m, 3H), $7.74(\mathrm{~s}, 1 \mathrm{H}) ;{ }^{13} \mathrm{C} \mathrm{NMR}\left(\mathrm{CDCl}_{3}\right)$ 17.38, $52.87,115.76,126.89,128.33,130.62,131.52,133.99$, $138.34,164.89$.

2h: $227 \mathrm{mg}(87 \%)$; mp 97-99 ${ }^{\circ} \mathrm{C}$; IR (KBr) 2238, 1716, $1632 \mathrm{~cm}^{-1} ;{ }^{1} \mathrm{H}$ NMR $\left(\mathrm{CDCl}_{3}\right) \delta 3.58(\mathrm{~s}, 2 \mathrm{H}), 3.89(\mathrm{~s}, 3 \mathrm{H})$, 3.93 (s, 3H), 3.94 (s, 3H), 6.94-6.97 (m, 2H), 7.05 (dd, 1H, J $=8.2$ and $1.8 \mathrm{~Hz}), 7.91(\mathrm{~s}, 1 \mathrm{H}) ;{ }^{13} \mathrm{C} \mathrm{NMR}\left(\mathrm{CDCl}_{3}\right) 17.02$, 52.62, 55.86, 55.99, 111.19, 112.19, 117.53, 119.62, 122.96, 126.36, 143.98, 149.06, 150.43, 166.44.

2i: $85 \mathrm{mg}(27 \%)$; $\mathrm{mp} 80-82^{\circ} \mathrm{C}$; IR (KBr) 2258, 1722, 
1658, 1536, $1354 \mathrm{~cm}^{-1} ;{ }^{1} \mathrm{H}$ NMR $\left(\mathrm{CDCl}_{3}\right) \delta 3.28(\mathrm{~s}, 2 \mathrm{H})$, $3.96(\mathrm{~s}, 3 \mathrm{H}), 7.58(\mathrm{~d}, 1 \mathrm{H}, J=8.9 \mathrm{~Hz}), 7.70(\mathrm{~s}, 1 \mathrm{H}), 7.87$ (d, $1 \mathrm{H}, J=8.9 \mathrm{~Hz}) ;{ }^{13} \mathrm{C} \mathrm{NMR}\left(\mathrm{CDCl}_{3}\right) 17.44,53.15,115.16$, $126.22,127.12$, 128.31, 128.85, 134.31, 136.66, 137.99, 147.26, 164.36.

Typical Procedure for the Preparation of Benzylidenesuccinic Acid 3a: A solution of $\mathrm{NaOH}(320 \mathrm{mg})$ in water (1 $\mathrm{mL})$ was added to a solution of $\mathbf{2 a}(201 \mathrm{mg}, 1.0 \mathrm{mmol})$ in $\mathrm{MeOH}(3 \mathrm{~mL})$, and the mixture was stirred and heated under reflux for $12 \mathrm{~h}$. The solvent was removed in vacuo. The aqueous layer was acidified with $2 \mathrm{~N}-\mathrm{HCl}$ and extracted with ether $(3 \times 25 \mathrm{~mL})$. The combined organic layer was dried over anhydrous $\mathrm{MgSO}_{4}$ and concentrated in vacuo to afford a solid, which was crystallized with EtOAc/hexane to give $150 \mathrm{mg}(73 \%)$ of $\mathbf{3 a}$; $\mathrm{mp} 186-187^{\circ} \mathrm{C}$ (lit. ${ }^{14 \mathrm{a}} 186-188^{\circ} \mathrm{C}$ ).

3b: $159 \mathrm{mg}(66 \%)$; mp $191-192{ }^{\circ} \mathrm{C}$ (lit. $\left.{ }^{14 \mathrm{~b}} 193-194{ }^{\circ} \mathrm{C}\right)$.

3c: $160 \mathrm{mg}(68 \%)$; mp $185-187^{\circ} \mathrm{C}$ (lit. $\left.{ }^{10 \mathrm{c}} 188-191{ }^{\circ} \mathrm{C}\right)$.

Typical Procedure for the Preparation of 4-Hydroxy-2naphthoic Acid 4a: Benzylidenesuccinic acid 3a (206 mg $1.0 \mathrm{mmol})$ was dissolved in conc. $\mathrm{H}_{2} \mathrm{SO}_{4}(464 \mathrm{mg})$ and stirred at room temperature for $8 \mathrm{~h}$. The reaction mixture was carefully poured over cold water $(1 \mathrm{~mL})$ and allowed to crystallize overnight at $5{ }^{\circ} \mathrm{C}$. The resulting crystals were filtered, washed with water, dried in vacuo and recrystallized from $\mathrm{EtOH} / \mathrm{H}_{2} \mathrm{O}$ to give $144 \mathrm{mg}$ (77\%) of $\mathbf{4 a}$; mp 223-224 ${ }^{\circ} \mathrm{C}$. (lit. ${ }^{10 \mathrm{a}} 225-226{ }^{\circ} \mathrm{C}$ ). IR (KBr) 3447, 1656, $1579 \mathrm{~cm}^{-1} ;{ }^{1} \mathrm{H}$ NMR (DMSO-d $\left.{ }_{6}\right) \delta 7.37(\mathrm{~s}, 1 \mathrm{H}), 7.58(\mathrm{~m}, 2 \mathrm{H}), 8.02(\mathrm{~m}, 1 \mathrm{H})$, 8.05 (s, 1H), $8.16(\mathrm{~m}, 1 \mathrm{H}), 10.49$ (s, 1H), 12.92 (br s, 1H); ${ }^{13} \mathrm{C}$ NMR (DMSO-d $\left.\mathrm{d}_{6}\right) 106.98,121.34,122.08,126.72$, 127.02, 127.06, 128.63, 129.08, 133.55, 153.43, 167.74.

4b: $155 \mathrm{mg}(70 \%)$; mp 295-297 ${ }^{\circ} \mathrm{C}$ (lit. ${ }^{10 \mathrm{~b}} 297^{\circ} \mathrm{C}$ ).

4c: $149 \mathrm{mg}(68 \%)$; mp $243-245^{\circ} \mathrm{C}$ (lit. ${ }^{10 \mathrm{c}} 243-246^{\circ} \mathrm{C}$ ).

Typical Procedure for the Preparation of Benzylidenesuccinimide 8a: To a stirred solution of cyanomethylpropenoate $2 \mathbf{a}(402 \mathrm{mg}, 2.0 \mathrm{mmol})$ in glacial HOAc $(10 \mathrm{~mL})$ was added $\mathrm{FeCl}_{3}$ (649 mg, $4.0 \mathrm{mmol}$ ), and the mixture was heated under reflux for $10 \mathrm{~h}$. After removal of the HOAc under vacuum, the residue was dissolved in $\mathrm{CHCl}_{3}(30 \mathrm{~mL})$, and the $\mathrm{CHCl}_{3}$ layer was washed with aq. $\mathrm{NaHCO}_{3}(20 \mathrm{~mL})$ and $\mathrm{H}_{2} \mathrm{O}(20 \mathrm{~mL})$, successively. The combined organic layer was dried over anhydrous $\mathrm{MgSO}_{4}$ and concentrated in vacuo to afford a solid, which was crystallized with $\mathrm{MeOH}$ to give $150 \mathrm{mg}(40 \%)$ of $\mathbf{8 a}$; mp 197-199 ${ }^{\circ} \mathrm{C}$. (lit. $\left.{ }^{11} 198-200{ }^{\circ} \mathrm{C}\right)$. IR (KBr) 3136, 1766, 1708, $1648 \mathrm{~cm}^{-1} ;{ }^{1} \mathrm{H} \mathrm{NMR}\left(\mathrm{CDCl}_{3}\right) \delta$ $3.64(\mathrm{~d}, 2 \mathrm{H}, J=2.4 \mathrm{~Hz}), 7.45-7.50(\mathrm{~m}, 5 \mathrm{H}), 7.62(\mathrm{t}, 1 \mathrm{H}, J=$ $2.4 \mathrm{~Hz}$ ), 8.19 (br s, $1 \mathrm{H}) ;{ }^{13} \mathrm{C}$ NMR (DMSO-d $)$ ) 34.75, 126.99, 129.00, 130.13, 131.38, 131.54, 134.14, 171.91, 175.70.

8b: 200 mg (45\%); mp 251-252 ${ }^{\circ} \mathrm{C}$ (lit. ${ }^{15 \mathrm{a}} 251-252^{\circ} \mathrm{C}$ ).

8c: $194 \mathrm{mg}$ (45\%); mp 246-247 ${ }^{\circ} \mathrm{C}$ (lit. ${ }^{15 \mathrm{~b}} 246.6-247.2^{\circ} \mathrm{C}$ ).

Acknowledgments. This work was supported by Hanyang University, Korea, made in the program year of 2004.

\section{References and Notes}

1. For reviews of the Baylis-Hillman reaction, see: (a) Drewes, S. E.; Roos, G. H. P. Tetrahedron 1988, 44, 4653. (b) Basavaiah, D.; Rao, P. D.; Hyma, R. S. Tetrahedron 1996, 52, 8001. (c) Ciganek,
E. In Organic Reactions; Paquette, L. A., Ed.; Wiley: New York, 1997; Vol. 51, p 201. (d) Langer, P. Angew. Chem., Int. Ed. 2000, 39, 3049. (e) Kim, J. N.; Lee, K. Y. Curr. Org. Chem. 2002, 6 , 627. (f) Basavaiah, D.; Rao, A. J.; Satyanarayana, T. Chem. Rev. 2003, 103, 811 .

2. (a) Genski, T.; Taylor, R. J. K. Tetrahedron Lett. 2002, 43, 3573. (b) Sammelson, R. E.; Gurusinghe, C. D.; Kurth, J. M.; Olmstead, M. M.; Kurth, M. J. J. Org. Chem. 2002, 67, 86. (c) Ciclosi, M.; Fava, C.; Galeazzi, R.; Orena, M.; Sepulveda-Arques, J. Tetrahedron Lett. 2002, 43, 2119

3. (a) Song, Y. S.; Lee, C. H.; Lee, K.-J. J. Heterocyclic Chem. 2003, 40, 939. (b) Lee, C. H.; Song, Y. S.; Cho, H. I.; Yang, J. W.; Lee, K.-J. J. Heterocyclic Chem. 2003, 40, 1103. (c) Basavaiah, D.; Reddy, R. M.; Kumaragurubaran, N.; Sharada, D. S. Tetrahedron 2002, 58, 3693. (d) Basavaiah, D.; Satyanarayana, T. Tetrahedron Lett. 2002, 43, 4301. (e) Kaye, P. T.; Nocanda, X. W. J. Chem. Soc., Perkin Trans. 1 2002, 1318. (f) Kim, J. N.; Kim, H. S.; Gong, J. H.; Chung, Y. M. Tetrahedron Lett. 2001, 42, 8341. (g) Kaye, P. T.; Nocanda, X. W. J. Chem. Soc., Perkin Trans. 1 2000, 1331. (h) Basavaiah, D.; Sreenivasulu, B.; Rao, J. S. Tetrahedron Lett. 2001, 42, 1147. (i) Alcaide, B.; Almendros, P.; Aragoncillo, C. Tetrahedron Lett. 1999, 40, 7537.

4. (a) Franck, X.; Figadère, B. Tetrahedron Lett. 2002, 43, 1449. (b) Iwabuchi, Y.; Sugihara, T.; Esumi, T.; Hatakeyama, S. Tetrahedron Lett. 2001, 42, 7867. (c) Iwabuchi, Y.; Furukawa, M.; Esumi, T.; Hatakeyama, S. Chem. Commun. 2001, 2030. (d) Mateus, C. R.; Feltrin, M. P.; Costa, A. M.; Coelho, F.; Almeida, W. P.; Jauch, J. Synlett 2001, 87. (e) Reiser, U.; Jauch, J. Synlett 2001, 90. (f) Jauch, J. Angew. Chem. Int. Ed. 2000, 39, 2764.

5. For a recent review of nitriles, see: Fleming, F. F.; Zhang, Z. Tetrahedron 2005, 61, 747

6. (a) Kim, J. N.; Lee, H. J.; Lee, K. Y.; Gong, J. H. Synlett 2002, 173. (b) Kim, J. N.; Lee, H. J.; Lee, K. Y.; Kim, H. S. Tetrahedron Lett. 2001, 42, 3737. (c) Basavaiah, D.; Kumaragurubaran, N. Tetrahedron Lett. 2001, 42, 477. (d) Im, Y. J.; Kim, J. M.; Mun, J. H.; Kim, J. N. Bull. Korean Chem. Soc. 2001, 22, 349. (e) Drewes, S. E.; Horn, M. M.; Ramesar, N. Synth. Commun. 2000, 30, 1045. (f) Foucaud, A.; El Guemmout, F. Bull. Soc. Chim. Fr. 1989, 403. (g) Basavaiah, D.; Pandiaraju, S. Tetrahedron 1996, 52, 2261. (h) Patra, A.; Roy, A. K.; Batra, S. Bhaduri, A. P. Synlett 2002, 1819. (i) Kim, J. N.; Im, Y. J.; Gong, J. H.; Lee, K. Y. Tetrahedron Lett. 2001, 42, 4195. (j) Kim, J. M.; Im, Y. J.; Kim, T. H.; Kim, J. N. Bull. Korean Chem. Soc. 2002, 23, 657. (k) Basavaiah, D.; Hyma, R. S.; Kumaragurubaran, N. Tetrahedron 2000, 56, 5905.

7. Chung, Y. M.; Gong, J. H.; Kim, T. H.; Kim, J. N. Tetrahedron Lett. 2001, 41, 9023.

8. Almeida, W. P.; Coelho, F. Tetrahedron Lett. 1998, 39, 8609.

9. (a) Hong, W. P.; Lee, K.-J. Synthesis 2005, 33. (b) Lee, C. H.; Lee, K.-J. Synthesis 2004, 1941. (c) Park, J. B.; Ko, S. H.; Kim, B. G.; Hong, W. P.; Lee, K.-J. Bull. Korean Chem. Soc. 2004, 25, 27. (d) Park, J. B.; Ko, S. H.; Hong, W. P.; Lee, K.-J. Bull. Korean Chem. Soc. 2004, 25, 927.

10. (a) Cason, J. J. Am. Chem. Soc. 1941, 63, 828. (b) El-Abbady, A. M.; Doss, S. H.; Moussa, H. H.; Nosseir, M. J. Org. Chem. 1961, 26, 4871. (c) House, H. O.; Larson, J. K. J. Org. Chem. 1968, 33, 448.

11. Hedaya, E.; Theodoropulos, S. Tetrahedron 1968, 24, 2241.

12. (a) Stobbe, H. Ber. 1893, 26, 2312. (b) Johnson, W. S.; Daub, G. H. In Organic Reactions, Vol. 6; Adams, R., Ed.; Wiley; New York, $1951 ; \mathrm{p} 1$

13. Wang, S.; Pan, J.; Hu, Y.; Hu, H. Synthesis 2005, 753.

14. (a) Jendralla, H.; Henning, R.; Seuring, B.; Herchen, J.; Kulitzscher, B.; Wunner, J. Synlett 1993, 155. (b) Mathur, K. B. L.; Krishnamurti, M.; Pandit, U. K. J. Am. Chem. Soc. 1953, 75, 3240.

15. (a) Vul'fson, N. S.; Zhurina, F. G.; Senyavina, L. B. Zh. Org. Khim. 1967, 3, 341; Chem. Abstr. 1967, 66, 115428. (b) Bryant, D. R.; Hauser, C. R. J. Am. Chem. Soc. 1961, 83, 3468. 